\title{
Efeito da atividade física programada sobre a aptidão física em escolares adolescentes
}

\author{
Effect of programmed physical activity on the physical fitness \\ of adolescent students
}

\author{
Edson dos Santos Farias \\ Wellington Roberto Gonçalves de Carvalho ${ }^{2}$ \\ Ezequiel Moreira Gonçalves 2.4 \\ Gil Guerra-Júnior ${ }^{2.4}$
}

1 Universidade Federal do Acre. Centro de Ciências da Saúde e Desporto. Rio Branco, AC. Brasil.

2 Universidade Estadual de Campinas. Programa de Pós-Graduação em Saúde da Criança e do Adolescente. Faculdade de Ciências Médicas. São Paulo, SP. Brasil.

3 Universidade Estadual de Londrina. Centro de Educação Física e Esporte. Grupo de Estudo e Pesquisa em Metabolismo, Nutrição e Exercício. Londrina, PR. Brasil.

4 Universidade Estadual de Campinas. Professor Livre-Docente. Departamento de Pediatria. Campinas, SP. Brasil.

Recebido em 16/03/09 Revisado em 01/05/09 Aprovado em24/07/09
Resumo - O estudo teve como objetivo verificar o efeito da atividade física programada sobre os testes de aptidão física em escolares adolescentes durante um ano de período letivo. Amostra foi composta de 383 alunos, divididos em dois grupos: caso, com 186 (96 meninos e 90 meninas) e controle, com 197 (108 meninos e 89 meninas), com idade entre 10 e 15 anos. Trata-se de estudo de intervenção com pré e pós-teste, com um ano de duração, no qual o grupo caso foi submetido à atividade física programada e o grupo controle com aulas convencionais de educação física escolar. A aptidão física foi avaliada por testes de sentar-e-alcançar (flexibilidade), resistência muscular (flexão e extensão do cotovelo) e aptidão cardiorrespiratória (corrida e caminhada de nove minutos). O desempenho motor verificado nos três testes analisados (flexibilidade, força e resistência) não apresentou melhora significativa quando envolveu a variável tempo do pré para o pós-teste, em ambos os grupos, mas quando comparado (tipo) o grupo caso (intervenção) com o controle, mostrou diferença significativa com melhora nos testes de força e resistência em ambos os sexos para o caso. Os meninos de ambos os grupos também mostraram predomínio nos testes de força e resistência. De uma forma geral, os valores foram maiores nos meninos em relação às meninas para resistência muscular e aptidão cardiorrespiratória e menor para flexibilidade, e todos aumentaram no pós-teste em relação ao pré-teste e foram maiores no grupo caso em relação ao controle. Em relação à flexibilidade, foi observada diferença estatisticamente significativa apenas entre os gêneros. Em relação à resistência muscular, após ajuste para idade e nível socioeconômico, foi observada diferença estatisticamente significativa entre os gêneros e entre os tipos. Em relação à aptidão cardiorrespiratória, foi observada diferença estatisticamente significativa entre os gêneros e entre os tipos.

Palavras-chave: Atividade motora; Saúde escolar; Aptidão física

Abstract - The objective of this study was to determine the influence of programmed physical activity on the physical fitness of adolescent students over one school year. The sample consisted of 383 students (age range: 10 to 14 years) divided into two groups: 186 cases (96 boys and 90 girls) and 197 controls (108 boys and 89 girls). An intervention study with pre-and post-tests was conducted, in which the intervention group was submitted to programmed physical activity, while the control group underwent conventional classes of school physical education. Physical fitness was assessed by sit-and-reach (flexibility), muscle endurance (elbow flexion and extension) and aerobic endurance (run/walk, 9 min) tests. Motor performance observed in the three tests (flexibility, strength and endurance) did not improve from pre-test to post-test in either group, but comparison of the intervention and control groups showed significant improvement in the strength and endurance tests for both genders in the intervention group. Boys of the two groups also showed dominance in the strength and endurance tests. In general, higher muscle strength and cardiorespiratory fitness and lower flexibility were observed for boys when compared to girls, and all parameters increased in the post-test and were higher in the intervention group compared to control A significant difference in flexibility was only observed between genders. With respect to muscle strength, a significant difference was observed between genders and between the intervention and control group after adjustment for age and socioeconomic level. Cardiorespiratory fitness differed significantly between genders and between the intervention and control group. Key words: Motor activity; School health; Physical fitness. 


\section{INTRODUÇÃO}

A prática de atividade física regular e a manutenção de níveis adequados de aptidão física têm sido reconhecidas por efeitos benéficos à saúde, sendo possível relacionar com a prevenção de doenças cardiovasculares, obesidade, diabetes, osteoporose, entre outras ${ }^{1-3}$.

Os componentes da aptidão física relacionada à saúde procuram abrigar atributos biológicos que possam oferecer alguma proteção ao aparecimento e ao desenvolvimento de distúrbios orgânicos induzidos por comprometimento da condição funcional; destacando entre eles, a capacidade cardiorrespiratória, força/resistência muscular e flexibilidade 4 .

Com aumento do sedentarismo e diminuição dos níveis de atividade física em crianças e adolescentes na fase escolar, a aptidão física se converteu em motivo de grande interesse para os profissionais na área de saúde $e^{5-7}$.

Para realmente alcançar as melhorias na aptidão física e os benefícios associados a esta, a atividade física deve partir de certos antecedentes, como os dados referentes à condição física pregressa, o conhecimento do comportamento da capacidade em relação à idade e ao gênero e a relação dos níveis de aptidão física em critérios de saúde 6 . Com esta finalidade, foram desenvolvidos instrumentos, testes e provas que permitem o acesso ao conhecimento das capacidades físicas e sua tendência, em especial, no âmbito escolar ${ }^{8,9}$.

Vários estudos mostram que, apesar dos jovens em idade escolar raramente apresentarem disfunções de ordem crônico-degenerativa, pouco se tem investido em sua formação, no que se refere à aderência de um estilo de vida mais ativo fisicamente. Em vista disso, Hussey et al. ${ }^{10}$ e Dubose et al. ${ }^{11}$ ressaltam que mesmo que os sintomas advindos das doenças crônico-degenerativas ainda não tenham se manifestado nessa fase, não significa que crianças e adolescentes estejam imunes aos fatores de riscos que no decorrer dos anos possam provocar estado de morbidez.

Portanto, este estudo teve como objetivo verificar o efeito da atividade física programada sobre os testes de aptidão física em escolares adolescentes, durante um ano de período letivo.

\section{PROCEDIMENTOS METODOLÓGICOS}

Trata-se de um estudo longitudinal de intervenção com pré e pós-teste. Foram incluídos no estudo os alunos matriculados que frequentavam regularmente as aulas de $5^{\mathrm{a}}$ a $8^{\mathrm{a}}$ séries do ensino fundamental no ano letivo de 2006. Foram excluídos do estudo os escolares com deficiências físicas permanentes ou temporárias que impossibilitassem participar dos testes de aptidão física e aqueles que se ausentaram em mais de $25 \%$ das aulas de educação física durante o estudo. No início do estudo, a amostra foi intencional, composta de 497 alunos, mas foi reduzida em função das intercorrências de ordem pessoal (perda $=53$ alunos) e da necessidade, ao final do estudo, de ajustar o tamanho da amostra para aumentar o poder estatístico discriminatório das variáveis (perda $=61$ alunos) (Tabela 1$)$.

Tabela 1: Valores dos tamanhos do efeito (delta) e do poder estatístico de discriminação das variáveis, analisadas por meio de análise de variância para medidas repetidas, ajustando para a idade e nível socioeconômico.

\begin{tabular}{lcc}
\hline Variável & Delta & Poder \\
\hline Flexibilidade & 1,77 & $99 \%$ \\
Corrida & 1,15 & $99 \%$ \\
Força & 0,75 & $99 \%$ \\
\hline
\end{tabular}

A amostra foi finalizada com 383 alunos, divididos em dois grupos: o grupo caso, constituído por 186 escolares, alunos do Colégio Adventista, sendo 96 meninos (12,4 $\pm 1,1$ anos) e 90 meninas $(12,2 \pm 1,1)$ e o grupo controle, alunos do Colégio Objetivo, num total de 197 escolares, 108 meninos $(12,6 \pm 1,2)$ e 89 meninas $(12,5 \pm 1,2)$, todos com idades entre 10 a 15 anos.

Ambos os grupos foram submetidos a duas aulas de educação física semanais, com duração de 60 minutos cada sessão, totalizando 684 aulas anuais. Os escolares do grupo-controle realizaram atividade física considerada habitual na escola, como recreação e jogos através de brincadeiras, exercícios de calistenia, aprendizagem de fundamentos das modalidades esportivas e jogos esportivos.

O nível de intensidade durante as atividades físicas foi controlado por monitores de frequência cardíaca da marca GEONAUTE CW 500.0, individuais e adequados às características fisiológicas de cada escolar. A intensidade foi baseada nas indicações do nível de atividade física adequado para melhor eficiência de queima lipídica, que compreende a faixa de $>55 \%$ da FCmáx. Os cálculos para se estimar a FC ideal para cada idade segue abaixo:

\footnotetext{
220 - idade do indivíduo = FCmáx de esforço

$\mathrm{FCmáx} \mathrm{de} \mathrm{esforço}-\mathrm{FC}$ de repouso $=\mathrm{FC}$ de reserva

$\mathrm{FC}$ de reserva $\times 0,55(55 \%)+\mathrm{FC}$ repouso $=\mathrm{FC}$ ideal $(55 \%)$ limite inferior

$\mathrm{FC}$ de reserva $\times 0,75(75 \%)+\mathrm{FC}$ repouso $=\mathrm{FC}$ ideal $(75 \%)$ limite superior
} 
Para melhor comparar e discutir os dados encontrados neste estudo, o nível de atividade física (NAF) em relação à FC foi classificado da seguinte forma, considerando a FC sub-máx. prevista (220 - idade):

- leve a moderada: FC entre 50 e $75 \%$

- moderada a intensa: FC > 75\% ou FC

Alguns autores, como Armstrong ${ }^{12}$ e Fernandez et al. ${ }^{13}$, recomendam a monitorização da frequência cardíaca (FC) como uma medida válida e prática para mensurar o nível habitual de atividade física em crianças e adolescentes.

O procedimento para medida da frequência cardíaca foi feito através de uma ficha de acompanhamento, com o seguinte critério: a cada 10 minutos, durante uma aula de 60 minutos, era registrada a frequência cardíaca do aluno que estava sendo monitorado, todos os alunos foram monitorados regularmente durante o decorrer do estudo. Durante as atividades, havia intervenção, quando necessário, para o controle da intensidade, com o objetivo de manter, o maior tempo possível, a turma se exercitando dentro da zona alvo individual > 50\% FCmáx.

As aulas foram compostas de três partes: a primeira, com atividade aeróbia (exercícios de flexibilidade, pular corda, caminhadas, corridas alternadas, saltos em ritmo contínuo, jogos recreativos) com duração 30 minutos; a segunda, com jogos esportivos (voleibol, futebol de salão, handebol e natação) com duração de 20 minutos; e a terceira, com alongamento, com duração 10 minutos.

Os grupos casos e controles foram semelhantes em relação à idade, ao gênero e ao nível socioeconômico (avaliado por meio de questionário, utilizando-se a classificação da Associação Brasileira de Empresas de Pesquisa - ABEP) ${ }^{14}$, adotou-se o seguinte critério de classificação: classe A (alta), B (média alta), C (média); o grau de instrução dos pais foi dividido em ensino fundamental $\left(1^{\mathrm{a}}\right.$ a $8^{\mathrm{a}}$ séries), médio completo e incompleto $\left(1^{\mathrm{a}}\right.$ a $3^{\mathrm{a}}$ séries) e superior completo e incompleto; moradores na residência de um a dois, três a cinco e mais de seis moradores; o grau de desenvolvimento puberal (verificado por autoavaliação, de acordo com o estadiamento de mamas para as meninas ${ }^{15} \mathrm{e}$ genitais para os meninos ${ }^{16}$. Neste estudo de coorte longitudinal, o ponto de corte para puberdade das meninas foi M2 (mamas) e os meninos PP2 (pêlos pubianos), a idade da menarca (IM) foi avaliada pelo método prospectivo através de duas perguntas diretas: a) Você já menstruou? Se sim, b) lembra o dia, mês e ano que menstruou pela primeira vez? Se a menina lembrasse, pelo menos, mês e ano, era considerado válido, caso contrário, era excluído do estudo. Foram consideradas maturadas as meninas com menstruação. $\mathrm{O}$ ponto de corte na Genitália IV foi adotado para maturação sexual dos meninos ${ }^{16}$.

Para estimar os níveis de atividade física (NAF), foi utilizado um Questionário Internacional de Atividade Física (IPAQ), versão curta, validado sua reprodutibilidade no Brasil por MATSUDO et al. ${ }^{17}$, indicado para o uso em adolescentes. Além da classificação do NAF (muito ativo, ativo, pouco ativo e sedentário), foram incluídas mais duas questões fechadas ao questionário: horas televisão e prática de esporte (tabela 3). O procedimento para aplicação dos questionários foi o mesmo, com a presença, em sala de aula, do pesquisador e do professor de sala que o auxiliou. Era lida a questão e o aluno (entrevistado) respondia e, quando necessário, o aluno podia levar o questionário para a casa para ser respondido pelos pais. Após, os questionários eram recolhidos para análise. As perguntas de ambos os questionários foram todas fechadas.

Os testes motores de aptidão física relacionada à saúde seguiram as recomendações propostas por AAHPERD $^{18}$ obedecendo à seguinte sequência: sentar e alcançar como indicador da flexibilidade; flexão e extensão do cotovelo como indicador de força ou resistência muscular; e corrida/caminhada de nove minutos como indicador da aptidão cardiorrespiratória. Os testes foram aplicados por uma equipe de avaliadores experientes e treinados previamente; um único avaliador foi responsável pelo registro das informações em cada teste, na tentativa de evitar erros aleatórios.

O estudo foi aprovado pelo Comitê de Ética em Pesquisa da Faculdade de Ciências Médicas da Universidade Estadual de Campinas (Unicamp) (Parecer $n^{\circ}$ 218/2005) e realizado em Porto Velho (RO), com a aprovação dos diretores das escolas e dos responsáveis pelos escolares envolvidos após assinatura do termo de consentimento livre esclarecido (TCLE).

\section{Análise estatística}

Para a determinação do poder estatístico da amostra analisada, utilizou-se um programa do software SAS denominado fpower. Este programa calcula o tamanho de amostra necessário para obter um determinado poder.

Para comparar as proporções, foram utilizados o teste qui-quadrado e o teste Exato de Fisher, quan- 
do necessário. Na comparação das medidas entre grupos e gêneros, foi utilizado a ANOVA (Análise de Variância). As análises foram corrigidas para a idade e nível socioeconômico.

O nível de significância adotado para os testes estatísticos foi de 5\%.

\section{RESULTADOS}

$\mathrm{Na}$ Tabela 2, são apresentados os resultados comparativos entre os grupos caso e controle em relação ao gênero, a maturação sexual, ao nível socioeconômico, ao grau de instrução dos pais e ao número de moradores na residência. Não foi observada diferença estatisticamente significativa em relação ao gênero, à maturação sexual, ao nível socioeconômico, ao grau de instrução dos pais e ao número de moradores na residência.
Os Valores de freqüência dos estadiamentos puberais e idade da observação dos mesmos são apresentados na Tabela 2, nos quais não foram observadas diferenças significativas estatisticamente, na comparação entre os grupos.

A Tabela 3 mostra os resultados comparativos entre os grupos caso e controle em relação aos níveis de atividade física. Não foi observada diferença estatisticamente significativa em relação ao nível de atividade física, ao número de horas diárias assistindo televisão e à prática de esporte.

Nos próximos resultados que serão apresentados, para facilitar a descrição dos dados, definiu-se as variáveis: tipo (grupos caso e controle), tempo (pré e pós-teste) e gênero (masculino e feminino).

A Tabela 4 mostra os dados das variáveis de flexibilidade, resistência muscular e aeróbia separados por gênero, tipo e tempo. De uma forma geral, os valores

Tabela 2: Análise descritiva e comparativa entre os grupos caso e controle, em relação às variáveis gêneros, maturação sexual, nível socioeconômico, grau de instrução dos pais e número de moradores na residência.

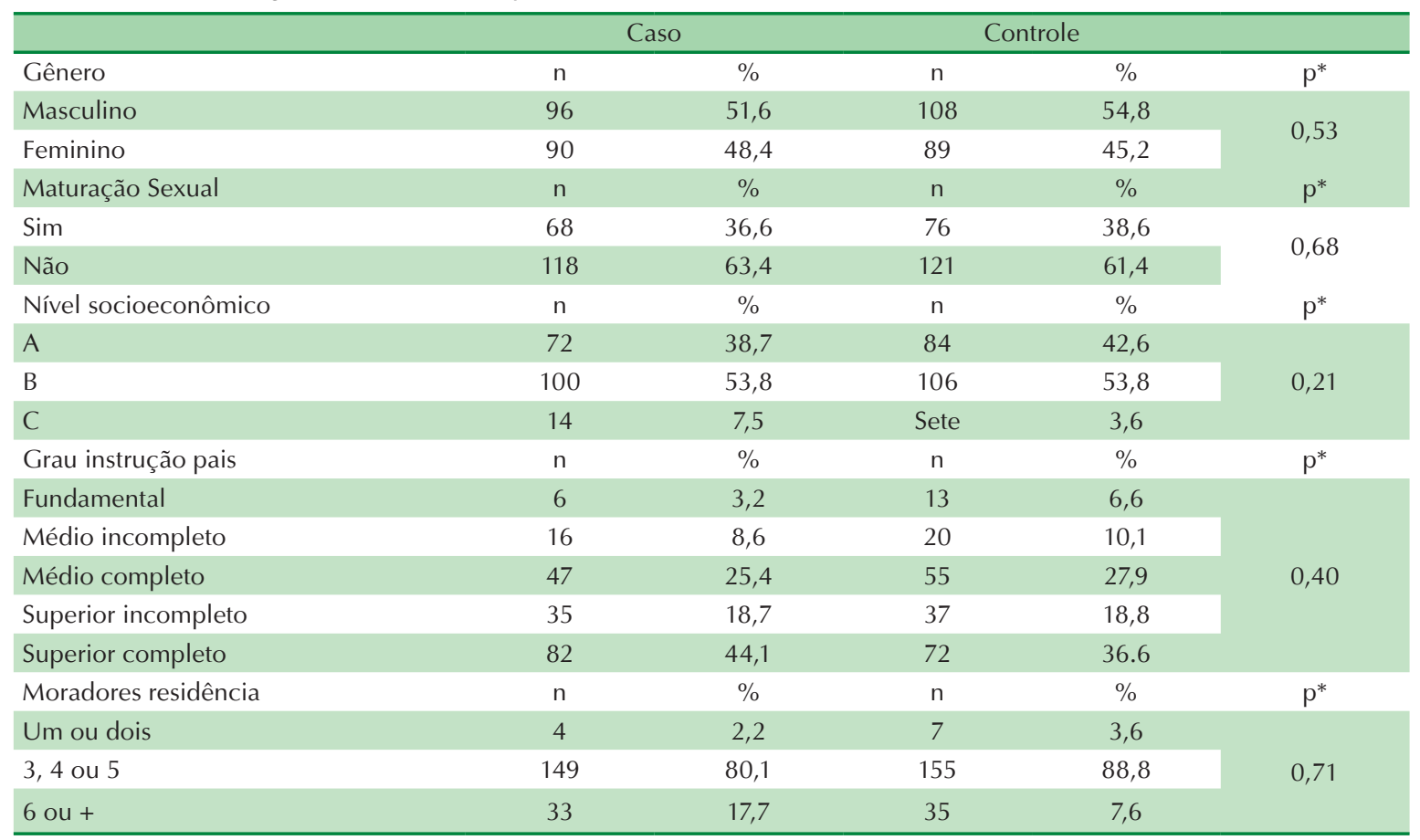

Tabela 3: Dados de puberdade entre os gêneros nos grupos caso e controle.

\begin{tabular}{lcccc}
\hline \multicolumn{2}{c}{ Casos } & \multicolumn{2}{c}{ Controles } \\
\hline Puberdade & Masculino (n) & Feminino $(\mathrm{n})$ & Masculino $(\mathrm{n})$ & Feminino $(\mathrm{n})$ \\
Estadio 1 & $11,2-11,6(30)$ & $11,0-11,7(27)$ & $10,8-11,2(29)$ & $10,8-11,3(18)$ \\
Estadio 2 & $11,8-12,5(29)$ & $11,7-12,1(32)$ & $12,2-12,6(35)$ & $11,0-11,6(39)$ \\
Estadio 3 & $13,1-13,4(27)$ & $12,9-13,3(21)$ & $13,2-13,7(29)$ & $12,8-13,2(18)$ \\
Estádio 4 & $14,1-14,5(10)$ & $13,9-14,2(8)$ & $14,0-14,3(15)$ & $13,6-13,9(9)$ \\
Estádio 5 & - & 13,8 e $14,7(2)$ & - & $13,8-14,3(5)$ \\
\hline
\end{tabular}

$\mathrm{IC}=$ intervalo de confiança de idade em cada estadio

(n) = número de sujeitos em cada estádio (383) 
Tabela 4: Análise descritiva e comparativa entre os grupos caso e controle, em relação ao nível de atividade física, ao número de horas diárias assistindo TV e à prática de esporte.

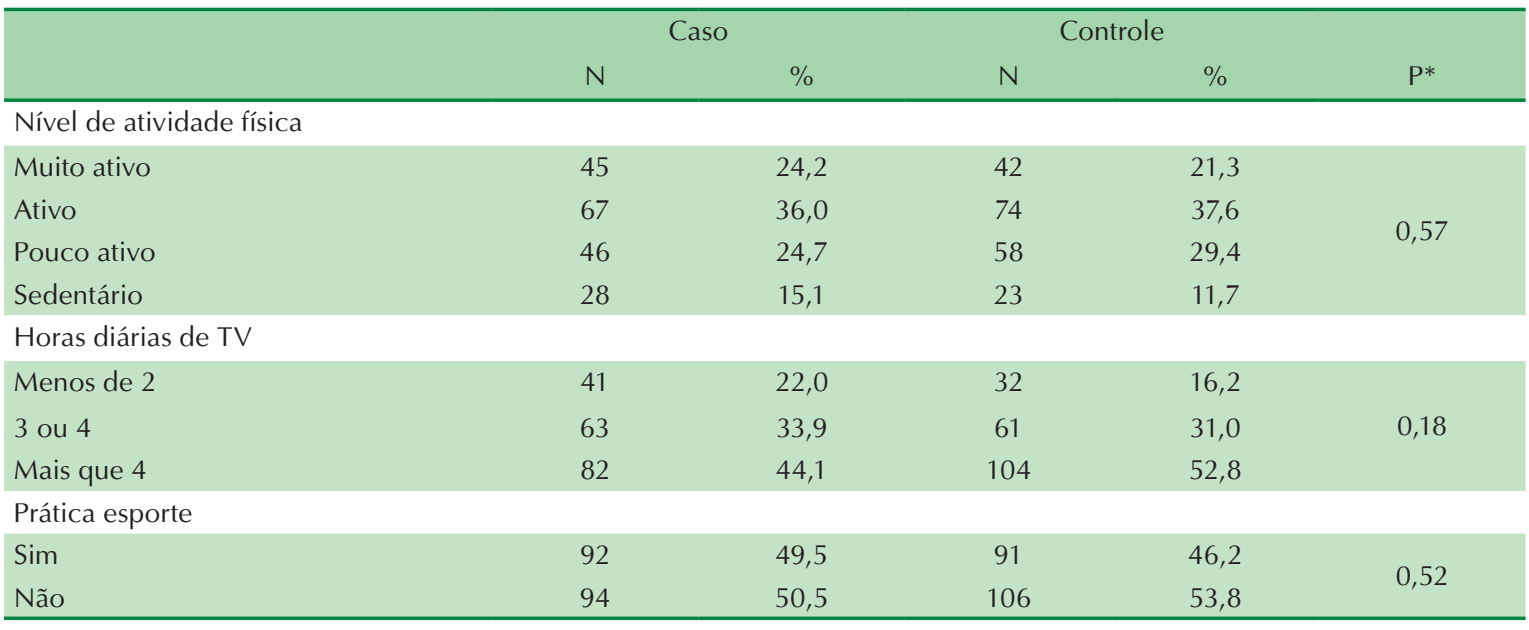

* Teste Qui-quadrado

Tabela 5: Dados de flexibilidade, resistência muscular (força) e aeróbia (corrida), em relação ao gênero do grupo caso e controle e ao pré e pós-teste.

\begin{tabular}{|c|c|c|c|c|c|c|c|c|c|}
\hline \multicolumn{6}{|c|}{ Casos } & \multicolumn{4}{|c|}{ Controles } \\
\hline & & \multicolumn{2}{|c|}{ Pré-teste } & \multicolumn{2}{|c|}{ Pós-teste } & \multicolumn{2}{|c|}{ Pré-teste } & \multicolumn{2}{|c|}{ Pós-teste } \\
\hline & & $\mathrm{M} \pm \mathrm{DP}$ & IC & $\mathrm{M} \pm \mathrm{DP}$ & IC & $M \pm D P$ & IC & $M \pm D P$ & IC \\
\hline \multirow{2}{*}{ Flex (cm) } & $M$ & $\begin{array}{l}20,5 \\
\pm 7,7\end{array}$ & $18,9-22,0$ & $\begin{array}{l}21,9^{c} \\
\pm 7,6\end{array}$ & $20,3-23,4$ & $\begin{array}{l}19,8 \\
\pm 7,6\end{array}$ & $18,3-21,2$ & $\begin{array}{l}21,0^{c} \\
\pm 7,7\end{array}$ & $19,6-22,5$ \\
\hline & $\mathrm{F}$ & $\begin{array}{l}22,0 \\
\pm 7,6\end{array}$ & $20,4-23,5$ & $\begin{array}{l}23,6^{c} \\
\pm 7,8\end{array}$ & $22,0-25,2$ & $\begin{array}{l}24,0 \\
\pm 8,5\end{array}$ & $22,2-25,8$ & $\begin{array}{l}25,8^{c} \\
\pm 9,2\end{array}$ & $24,0-27,8$ \\
\hline \multirow{2}{*}{ Força № rep. } & $M$ & $\begin{array}{r}13,9 \\
\pm 8,6\end{array}$ & $12,2-15,6$ & $\begin{array}{c}15,7 \mathrm{~b}, \mathrm{c} \\
\pm 9,0\end{array}$ & $13,9-17,5$ & $\begin{array}{l}11,3 \\
\pm 7,7\end{array}$ & $9,8-12,8$ & $\begin{array}{l}11,8 \\
\pm 8,1\end{array}$ & $10,2-13,3$ \\
\hline & $\mathrm{F}$ & $\begin{array}{c}7,0 \\
\pm 4,4\end{array}$ & $6,1-8,0$ & $\begin{array}{l}7,7^{\mathrm{b}, \mathrm{c}} \\
\pm 4,8\end{array}$ & $6,7-8,7$ & $\begin{array}{c}5,6 \\
\pm 4,4\end{array}$ & $4,7-6,5$ & $\begin{array}{l}5,5^{b, c} \\
\pm 5,3\end{array}$ & $4,4-6,6$ \\
\hline \multirow{2}{*}{ Corrida $\left(\mathrm{m} / 9^{\prime}\right)$} & $M$ & $\begin{array}{r}1216 \\
\pm 222\end{array}$ & $1171,0-1261$ & $\begin{array}{c}1311 \text { b,c } \\
\pm 258\end{array}$ & $\begin{array}{c}1258- \\
1363\end{array}$ & $\begin{array}{l}1128 \\
\pm 252\end{array}$ & $\begin{array}{c}1080- \\
1177\end{array}$ & $\begin{array}{c}1118^{\mathrm{b}, \mathrm{c}} \\
\pm 262\end{array}$ & 1068-1168 \\
\hline & $\mathrm{F}$ & $\begin{array}{l}1054 \\
\pm 171\end{array}$ & 1019-1090 & $\begin{array}{c}1126^{b, c} \\
\pm 191\end{array}$ & $\begin{array}{c}1086- \\
1166\end{array}$ & $\begin{array}{c}915 \\
\pm 163\end{array}$ & $881-949$ & $\begin{array}{c}909 \text { b,c } \\
\pm 166\end{array}$ & $874-944$ \\
\hline
\end{tabular}

$M=$ masculino; $F=$ feminino; $p<0,05(a=$ tempo, $b=$ tipo, $c=s e x o$ )

ANOVA para medidas repetidas, com ajuste para idade e nível socioeconômico

foram maiores nos meninos em relação às meninas para resistência muscular e aeróbia e menor para flexibilidade, e todos aumentaram no pós-teste em relação ao pré-teste e foram maiores no caso em relação ao controle. Em relação à flexibilidade, foi observada diferença estatisticamente significativa apenas entre os gêneros. Em relação à resistência muscular, após ajuste para idade e nível socioeconômico, foi observada diferença estatisticamente significativa entre os gêneros e entre os tipos. Em relação à resistência aeróbia, foi observada diferença estatisticamente significativa entre os gêneros e entre os tipos.

\section{DISCUSSÃO}

Os programas regulares de atividade física estão sendo estudados mais criteriosamente somente nos últimos anos. No entanto, diversos estudos, ao longo do tempo, têm buscado investigar o efeito desse tipo de treinamento para melhoria de diferentes componentes da aptidão física ${ }^{19}$.

Os resultados observados, após ajuste para idade e nível socioeconômico, antes e após o período de intervenção, mostraram manutenção nas variáveis da aptidão física, com tendência de melhora na aptidão física verificada no grupo caso e não se confirmando no grupo controle, em especial, nos testes de força e resistência.

Em adolescentes, vale destacar que nem sempre as alterações ocorrem de forma tão sensível, em razão das adaptações metabólicas geradas durante o processo de treinamento e, principalmente, devido às alterações de crescimento e composição corporal próprias do estirão de crescimento e da maturação sexual ${ }^{20}$. 
Os resultados do estudo revelaram que o grupo caso obteve superioridade sobre o controle, nas variáveis de resistência muscular e aeróbia. Os escolares do grupo caso foram submetidos a uma atividade física que envolveu as capacidades aeróbia e anaeróbia, em conjunto com outras qualidades físicas (força, agilidade) durante as aulas de Educação Física Escolar. No resultado da flexibilidade, após ajuste para idade e nível socioeconômico, observou-se que houve melhora nos grupos do pré e pós-teste, com significância para o gênero feminino que apresentou maiores escores para o teste.

O teste de "sentar-e-alcançar" por meio do qual é exigida a participação da flexibilidade de várias articulações simultaneamente, verifica-se que as meninas mostram tendências, em todas as idades, de apresentarem valores médios significativos superiores em relação aos meninos.

Silva et $\mathrm{al}^{21}$ e Araújo e Oliveira ${ }^{22}$ mostraram que há aumento da flexibilidade para as meninas, contra uma diminuição para os meninos, com o avanço do período pubertário. Em relação a esta diminuição da flexibilidade nos meninos, Philippaerts et al. ${ }^{23}$ observaram, ainda, que ela pode ocorrer antes mesmo do pico de velocidade de crescimento, estabilizando-se logo após esse período. Observando este aspecto para ambos os gêneros, não houve melhoria significativa dos níveis de flexibilidade, envolvendo a variável tempo (pré e pós-teste).

O teste de resistência muscular, puxada em suspensão na barra "modificada", mostrou diferenças estatisticamente significativas, com valores superiores nos meninos em relação às meninas e no grupo caso em relação ao controle. Esses resultados podem se justificar pelo fato do advento da puberdade nos meninos provocar um ganho de massa muscular bastante acentuado, em consequência de uma maior produção de hormônios andrógenos ${ }^{4}$.

Todavia, paralelamente ao aumento da massa muscular, ocorre, também, maior acúmulo de tecido adiposo nas meninas que, por sua vez, não deverá contribuir na realização de movimentos que envolvem a força e a resistência muscular, contudo, provocará aumentos significativos no peso corporal ${ }^{24}$.

Enquanto os meninos alcançam os valores máximos em força relativa, na idade adulta, entre as meninas o pico máximo da força relativa ocorre do início da puberdade ${ }^{25}$. Outro aspecto relevante nesta faixa etária é que enquanto os meninos preferem atividades mais intensas de corrida, velocidade e força, as meninas preferem atividades leves e moderadas, como exercícios de alongamento, flexibilidade, coordenação, que envolvam o mínimo de força possível ${ }^{12,26}$.
Com relação ao teste de aptidão cardiorrespiratório, os resultados também foram significativamente superiores no grupo caso e entre os meninos.

Tomkinson e Olds ${ }^{7}$ enfatizam que, durante esta faixa etária, juntamente com o rápido crescimento somático, e consequente aumento da massa muscular que está intimamente ligado ao consumo de oxigênio, há o aumento de órgãos como o coração e os pulmões que têm influência direta na melhora da resistência cardiorrespiratória, principalmente, por melhorar a captação e utilização do oxigênio.

Hansen et al..$^{27}$ colocam que o exercício melhora a habilidade para aumentar a oxidação de gordura em 24 horas, em resposta ao aumento da ingestão de gordura na dieta. Dessa forma, o exercício pode contribuir com a redução do balanço positivo de gordura e em consequência, na redução do ganho de peso.

Assim sendo, os fatores biológicos que podem ter contribuído para que se possam observar diferenças entre os gêneros quanto à resistência geral é resumida da seguinte forma: a) maior ganho de força associado ao aumento na secreção de hormônios andrógenos na puberdade entre os meninos; b) acúmulo maior de gordura entre as meninas na puberdade; c) pequena vantagem no tamanho corporal, traduzida pela estatura, que ocorre entre os meninos; d) vantagens anatômicas específicas dos meninos, como maior comprimento de pernas e tipo de quadril mais apropriado, favorecendo o sistema de alavancas; e) vantagens na função fisiológica entre os meninos, favorecendo eficiência dos sistemas de produção de energia.

Os níveis de aptidão física de crianças e adolescentes, além da influência das transformações fisiológicas e anatômicas decorrentes das descargas hormonais que são aumentadas com a chegada da puberdade são influenciados pela quantidade de atividade física habitual, que de acordo com Ronque et al. ${ }^{28}$, declinam claramente da infância para a adolescência e para o adulto. As aulas de Educação Física Escolar podem priorizar a inclusão de atividades que desenvolvam a aptidão física dos seus alunos, principalmente, as relacionadas à saúde, pois conforme Pate ${ }^{29}$, seus componentes são mais suscetíveis aos fatores ambientais, enquanto os componentes relacionados ao desempenho são mais influenciados pela hereditariedade.

Entretanto, conforme Farinatti e Ferreira ${ }^{30}$, o que se vê nos programas de Educação Física Escolar é um predomínio do jogo e de atividades lúdicas, que acabam por privilegiar de forma exagerada as capacidades motoras coordenativas. Por outro lado, 
verifica-se certa relutância e indisponibilidade para aplicar programas onde as capacidades motoras condicionais, entre as quais estão inseridas a força/ resistência muscular e a resistência cardiorrespiratória, sejam solicitadas. É importante que a aptidão física relacionada à saúde seja alvo de mais atenção nas aulas de educação física escolar.

Cabe ressaltar que o estudo teve suas limitações como: o instrumento utilizado para estimar a frequência cardíaca (ambiente, temperatura, etc.) durante as aulas, a frequência duas vezes por semana de aula, a resistência dos alunos na prescrição de trabalho de força e corrida, o teste cardiorrespiratório foi adaptado à quadra. Apesar dessas considerações, estamos cientes das limitações deste estudo em relação aos problemas citados acima, que envolveu um protocolo árduo e um tempo longo de aulas monitoradas. No entanto, no decorrer do período de intervenção, os alunos, gradativamente, foram mudando seu estilo de vida, melhorando sua aderência à prática da atividade física nas aulas, com melhor participação e aceitabilidade aos exercícios que envolvem maior força e resistência. Provavelmente, se não ocorreu significância do pré para o pós-teste na variável tempo, pelo menos, o grupo de intervenção obteve resultados melhores e significativos em relação ao grupo controle.

Considerando as limitações do estudo, destaca-se a importância de incentivar os professores de educação física a incluirem os testes de aptidão física relacionado à saúde como programa nos currículos escolares, como um meio de incentivar e motivar a prática da atividade física nas aulas de educação física escolar.

\section{CONCLUSÃO}

Considerando os resultados apresentados, percebese que o desempenho motor verificado nos três testes analisados (flexibilidade, força e cardiorrespiratório) não apresentou melhora significativa quando envolveu a variável tempo do pré para o pós-teste, em ambos os grupos, mas quando comparado (tipo) o grupo caso (intervenção) com o controle, mostrou diferença significativa com melhora nos testes de força e resistência em ambos os sexos para o caso. Os meninos de ambos os grupos também mostraram predomínio nos testes de força e resistência.

\section{REFERÊNCIAS BILIOGRÁFICAS}

1. Vainionpaa A, Korrpelainen R, Haikkonen H, Knip M, Leppaluoto AMD, Jamsa T. Effect pf impact exercise on physical performance and cardiovascular risk factors. Med Sci Sports Exerc 2007;39(5):756-763.
2. Nishijima H, Stake K, Igarashi K, Morita N, Kanazawa $\mathrm{N}$, Okita K. Effects of exercise in overweight Japanese with multiple cardiovascular risk factors. Med Sci Sports Exerc 2007;39(6):926-933.

3. Singh AS, Paw MJ, Brug J, Van Mechelen WV. Short-term effects of school-based weight gain prevention among adolescents. Arch Pediatr Adoles Med 2007;161(6):656-671.

4. Malina RM. Physical fitness of children and adolescents in the United States: status and secular change. Med Sport Sci 2007;50:67-90.

5. Mojica GT, Poveda JG, Pinilla MI, Lobelo, F. Relationship between overweight, physical activity and fitness in school-aged boys in Bogotá. Arch Latino Am Nutr 2008;58(3):265-273.

6. Cleland V, Dwyer T, Blizzard L, Venn A. The provision of compulsory school physical activity: associations with physical activity, fitness and overweight in childhood and twenty years later. Int J Behav Nutr Phys Act 2008; 5:14.

7. Tomkinson GR, Olds TS. Secular changes in aerobic fitness test performance of Australian children and adolescents. Med Sport Sci 2007;50:168-182.

8. Bayer O, Bolte G, Morlock G, Ruckinger S, Von Kries R, Fromme H. et al. A simple assessment of physical activity is associated with obesity and motor fitness in pre-school children. Public Health Nutr, 2008;12(8):1242-1247.

9. Morrow JR Jr, Fulton JE, Brener ND, Kohl HW. Prevelence and correlates of physical fitness testing in V. S. Schools - 2000. Res Q Exerc Sport 2008;79(2):141-148.

10. Hussey J, Bell C, Bennett K, O’Dwyer J, Gormely, J. Relationship between the intensity of physical activity, inactivity, cardiorrespiratory fitness and body composition in 7-10-year-old Dublin children. Br J Sports Med 2007;41(5):311-316.

11. Dubose KD, Eisenmann JC, Donnelly JE. Aerobic fitness attenuates the metabolic syndrome score in normal-weight, at-risk-for-overweight, and overweight children. Pediatrics 2007;120(5):1262-1268.

12. Armstrong N. Aerobic fitness of children and adolescents. J Pediatr 2006;82(6):406-408.

13. Fernandez AC, Mello MT, Tufik S, Castro PM, Fisberg M. Influência do treinamento aeróbio e anaeróbio na massa de gordura corporal de adolescentes obesos. Rev Bras Med Esporte 2004;10(3):152-158.

14. Associação Nacional de Empresas de Pesquisa (ABEP). Critério de classificação econômica - Brasil. Disponível em: http://www.anep.org.br/mural/anep/04-12-97-cceb. htm. [2007 mai 18].

15. 5. Marshall WA, Tanner SM. Variations in the pattern of puberal changes in girls. Arch Dis Child. 1969;44(235):291-303.

16. Marshall WA, Tanner SM. Variations in the pattern of puberal changes in boys. Arch Dis Child 1970;45(239):13-23.

17. Matsudo SM, Araújo T, Matsudo VM, Andrade D, Andrade E, Oliveira LC. Questionário internacional de atividade física (IPAQ): estudo de validade e reprodutibilidade no Brasil. Rev Bras Ativ Física Saúde 2001;6 (2):05-18. 
18. Canadian association for health, physical education, recreation and dance. The cahperd Fitness-performance II test manual. Vanier, Cahperd, 1980.

19. American College Of Sports Medicine. Physical activity and bone health. Med Sci Sport Exerc 2004;36(11):1985-1996,

20. Parent AS, Teilmann G, Juul A, Skakkebaek NE, Toparri J, Bourguignon JP. The timing of normal puberty and the age limits of sexual precocity: variations around the world, secular trends, and changes after migration. Endocr Rev. 2003; 24(5):668-693.

21. Silva DJL, Santos JAR, Oliveira BMPM. A flexibilidade em adolescentes - um contributo para a avaliação global. Rev Bras Cineantropom Desempenho Hum 2006;8(1):72-79.

22. Araújo SS, Oliveira ACC. Aptidão física em escolares de Aracaju. Rev Bras Cineantropom Desempenho Hum 2008;10(3):271-276.

23. Philippaerts RM, Vaeyens R, Janssens M, Renterghem RV, Marthys D, Craen R et al.. The relationship between peak height velocity and physical performance in youth soccer players. J Sports Sci 2006;24(3):221- 230.

24. Mikkelsson LO, Nupponen H, Kaprio J, Kautiainen H, Mikkelsson M, Kujala UM. Adolescent flexibility, endurance strength, and physical activity as predictors of adult tension neck, low back pain, and knee injury: a 26 year follow up study. Br J Sports Med 2006;40(2):107-113.

25. Pereira IRP, Gomes PSC. Testes de força e resistência muscular: confiabilidade e predição de uma repetição máxima - revisão e novas evidências. Rev Bras Med Esporte 2003;9(5):325-335.
26. Perry MC, Straker LM, O'sullivan PB, Smith AJ, Hands B. Fitness motor competence and body composition as correlates of adolescent, neck/shoulder pain: an exploratory cross-section study. BMC Public Health 2008;15(8) 290-299.

27. Hansen KC, Zhang Z, Gomez T, Adams A, Schoeller DA. Exercise increases the proportion of fat utilization during short-term consumption of a high-fat diet. Am J Clin Nutr 2007;85(1):109-116.

28. Ronque ERV, Cyrino, ES, Dórea V, Serassuelo Júnior $\mathrm{H}$, Galdi EHG, Arruda M. Diagnóstico da aptidão física em escolares de alto nível socioeconômico: avaliação referenciada por critérios de saúde. Rev Bras Med Esporte 2007;13 (2):71-76.

29. Pate RR. The evolving definition of physical fitness Quest 1988;40(3):174-179.

30. Farinatti P, Ferreira M. Saúde, promoção da saúde e educação física. Rio de Janeiro: UERJ; 2006.
Endereço para correspondência
Edson dos Santos Farias
Universidade Federal do Acre
Centro de Ciência da Saúde e Desporto
Campus Universitário - Rodovia BR 364, Km 04, $n^{\circ} 6637$ - Distrito Industrial. Caixa Postal 500
Cep: 69915-900 Rio Branco. AC
E-mail: esfarias@bol.com.br 